RESEARCH HIGHLIGHTS

\title{
Coordinated defence
}

An important link between the two defence systems that plants use to detect and combat microbial pathogens has been reported in Cell.

Innate immune systems in plants and animals detect pathogen-associated molecular patterns (PAMPs), which are pathogen-specific molecules such as bacterial lipopolysaccharide. In animals, Toll-like receptors (TLRs) detect PAMPs, whereas in plants, the innate immune response (basal defence) is mediated by PAMP receptors. Following PAMP detection, receptors activate signalling pathways that effect defence responses.

A second system in plants recognizes bacterial virulence factors (type III effectors) that are injected into plant cells by the type III secretion system. When a resistance $(\mathrm{R})$ protein that recognizes a specific type III effector is present, a defence response that includes cell death, named the hypersensitive response (HR), occurs and the type III effector is known as an avirulence protein (Avr). If the $\mathrm{R}$ protein is absent, there is no HR and the type III effector mediates its virulence function. Some R proteins recognize interactions between type III effectors and cellular proteins instead of interacting with the type III effector. Such R proteins 'guard' the cellular protein that is targeted by the type III effector - the basis of the guard hypothesis.

Here, Kim et al. investigated how pathogen Avr proteins affect plant defences using a model system of
Pseudomonas syringae infection of Arabidopsis thaliana. The $P$. syringae type III effector AvrRpm1 phosphorylates A. thaliana RIN4, and the R-protein guard RPM1 detects RIN4 P. A second P. syringae type III effector, AvrRpt2, degrades RIN4, which activates the R-protein guard, RPS2. Using molecular and cellular approaches, Kim et al. showed that both AvrRpm1 and AvrRpt 2 inhibit PAMP-induced defences. Plus, overexpression of RIN4, the target of these bacterial proteins, also inhibited the PAMP response. As plants lacking RIN4 had increased PAMP responses, RIN4 is a bona fide negative regulator of the PAMP response.

However, the plant is not easily outsmarted by its pathogen and uses the R-protein guards RPM1 and RPS2 to oversee RIN4. If RIN4 functions are perturbed, a second line of defence is activated by the R-protein

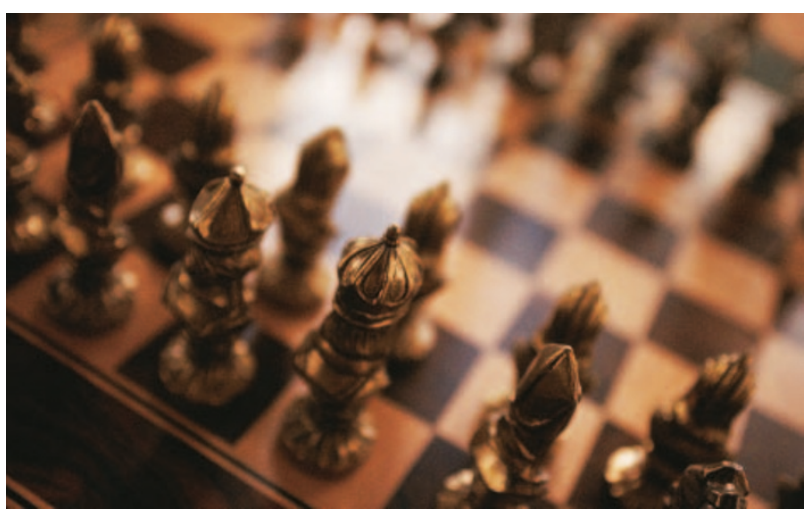

guards. This is the first study to show a link (RIN4) between the induction of PAMP responses and the R-protein-mediated defence system, which indicates that plants coordinate their defences - the plant uses RPM1 and RPS2 to guard against pathogens that perturb the PAMP-signalling system.

The authors speculate that their findings might have parallels in animal immunity. A Salmonella enterica sv. Typhimurium protein AvrA inhibits innate immune responses in animals and can also induce apoptosis, akin to the induction of the HR in plants by bacterial virulence proteins - so perhaps animals also use guard proteins.

\section{(2) References and links} ORIGINAL RESEARCH PAPER Kim, M.G. et al. Two Pseudomonas syringae type Ill effectors inhibit RIN4-regulated basal defense in Arabidopsis. Cell 121, 749-759 (2005) 